\title{
Simplified pure spinor $b$ ghost in a curved heterotic superstring background
}

\section{Nathan Berkovits ${ }^{a}$ and Osvaldo Chandia ${ }^{b}$}

${ }^{a}$ ICTP South American Institute for Fundamental Research, Instituto de Física Teórica, UNESP - Universidade Estadual Paulista, Rua Dr. Bento T. Ferraz 271, 01140-070, São Paulo, SP, Brasil

${ }^{b}$ Departamento de Ciencias, Facultad de Artes Liberales, Facultad de Ingeniería y Ciencias, Universidad Adolfo Ibáñez, Diagonal Las Torres 2640, Peñalolén, Santiago, Chile

E-mail: nberkovi@ift.unesp.br, ochandiaq@gmail.com

ABSTRACT: Using the RNS-like fermionic vector variables introduced in arXiv:1305.0693, the pure spinor $b$ ghost in a curved heterotic superstring background is easily constructed. This construction simplifies and completes the $b$ ghost construction in a curved background of arXiv:1311.7012.

Keywords: Superstrings and Heterotic Strings, Conformal Field Models in String Theory

ARXiv EPRINT: 1403.2429 


\section{Contents}

1 Introduction 1

2 Review of the non-minimal pure spinor formalism 2

2.1 Non-minimal pure spinor formalism in flat space 2

2.2 Non-minimal pure spinor formalism in a curved background 4

3 Definition of $\overline{\boldsymbol{\Gamma}}_{\boldsymbol{a}}$ in curved background $\quad 6$

$\begin{array}{lll}3.1 & \text { Simplified BRST transformations } & 6\end{array}$

$\begin{array}{ll}3.2 \text { Construction of } \bar{\Gamma}_{a} & 7\end{array}$

4 Definition of $b$ ghost in curved background $\quad 8$

$\begin{array}{ll}\text { A Computations in a flat background } & 9\end{array}$

\section{Introduction}

Although the pure spinor formalism for the superstring [1] has several nice features such as manifest spacetime supersymmetry and a simple BRST operator, a complicated feature of the formalism which needs to be better understood is the pure spinor $b$ ghost. Unlike in the bosonic string or Ramond-Neveu-Schwarz (RNS) string where the $b$ ghost is a fundamental worldsheet variable, the $b$ ghost in the pure spinor formalism is a composite operator constructed from the other worldsheet variables.

The BRST operator $Q$ in the pure spinor formalism is independent of the target-space background and takes the simple form

$$
Q=\int d z\left(\lambda^{\alpha} d_{\alpha}+\widehat{\omega}^{\alpha} r_{\alpha}\right)
$$

where $\left(\lambda^{\alpha}, d_{\alpha}, \widehat{\omega}^{\alpha}, r_{\alpha}\right)$ are fundamental worldsheet variables. To satisfy the relation $\{Q, b\}=T$ where $T$ is the conformal stress tensor, $b$ was constructed in a flat background in [2] as a complicated function of the worldsheet fields. This construction of the pure spinor $b$ ghost was simplified in [3] by introducing a fermionic vector field $\bar{\Gamma}_{a}$ which was related to the usual RNS fermionic vector field $\psi_{a}$ by "dynamical twisting".

An important question is how to generalize this construction of the pure spinor $b$ ghost in a curved superstring background. In [4], the pure spinor $b$ ghost was constructed in a super-Maxwell background of the open superstring, and in [5], the pure spinor $b$ ghost was constructed in an $\mathrm{N}=1$ supergravity background of the heterotic superstring. The construction in [5] for a curved heterotic superstring background was quite complicated and some of the coefficients of the composite operator for the pure spinor $b$ ghost were not explicitly computed. 
In this paper, we will use the RNS-like fermionic vector field $\bar{\Gamma}_{a}$ of [3] to simplify the construction of the pure spinor $b$ ghost in a curved heterotic superstring background. When expressed in terms of $\bar{\Gamma}_{a}$, the composite operator for the pure spinor $b$ ghost in a curved heterotic background is a simple covariantization of the composite operator in a flat background that was found in [3]. In addition to simplifying the construction in a curved heterotic background, it is hoped that this method involving $\bar{\Gamma}_{a}$ will also be useful for constructing the pure spinor $b$ ghost in an $\mathrm{N}=2$ supergravity background of the Type II superstring.

In section 2, we review the pure spinor formalism of the heterotic superstring in a flat and curved target-superspace background. In section 3, we define the RNS-like variable $\bar{\Gamma}_{a}$ in a curved background. And in section 4 , we use $\bar{\Gamma}_{a}$ to explicitly construct the pure spinor $b$ ghost in a curved heterotic superstring background. The appendix computes the BRST transformations of $\bar{\Gamma}_{a}$ and the $b$ ghost in a flat background.

\section{Review of the non-minimal pure spinor formalism}

In this section we review the non-minimal pure spinor formalism of the heterotic superstring in flat space [2] and in a curved background [5].

\subsection{Non-minimal pure spinor formalism in flat space}

The non-minimal pure spinor formalism in the heterotic superstring is constructed using the ten-dimensional $N=1$ superspace coordinates $\left(X^{a}, \theta^{\alpha}\right)$ for $a=0$ to 9 and $\alpha=1$ to 16 , the conjugate variable of $\theta^{\alpha}$ which is called $p_{\alpha}$, a set of pure spinor variables $\left(\lambda^{\alpha}, \widehat{\lambda}_{\alpha}, r_{\alpha}\right)$ together with their conjugate variables $\left(\omega_{\alpha}, \widehat{\omega}^{\alpha}, s^{\alpha}\right)$, and the same 32 fermionic right-moving variables $\xi^{R}$ for $R=1$ to 32 as in the RNS heterotic superstring formalism. The pure spinor variables are constrained to satisfy

$$
\left(\lambda \gamma^{a} \lambda\right)=\left(\widehat{\lambda} \gamma^{a} \widehat{\lambda}\right)=\left(\widehat{\lambda} \gamma^{a} r\right)=0
$$

where $\left(\gamma^{a}\right)_{\alpha \beta}$ and $\left(\gamma^{a}\right)^{\alpha \beta}$ are the symmetric gamma matrices which satisfy the Dirac algebra

$$
\left(\gamma^{a}\right)_{\alpha \gamma}\left(\gamma^{b}\right)^{\gamma \beta}+\left(\gamma^{b}\right)_{\alpha \gamma}\left(\gamma^{a}\right)^{\gamma \beta}=2 \eta^{a b} \delta_{\alpha}^{\beta}
$$

Because of the pure spinor conditions (2.1), the conjugate pure spinor variables are defined up to the gauge invariances

$$
\delta \omega_{\alpha}=\left(\lambda \gamma^{a}\right)_{\alpha} \Lambda_{1 a}, \quad \delta s^{\alpha}=\left(\gamma^{a} \widehat{\lambda}\right)^{\alpha} \Lambda_{2 a}, \quad \delta \widehat{\omega}^{\alpha}=\left(\gamma^{a} \widehat{\lambda}\right)^{\alpha} \Lambda_{3 a}-\left(\gamma^{a} r\right) \Lambda_{2 a},
$$

where $\Lambda_{1}, \Lambda_{2}, \Lambda_{3}$ are arbitrary gauge parameters. The action of the theory is quadratic in these variables and is given by

$$
S=S_{0}+\int d^{2} z\left(\widehat{\omega}^{\alpha} \widehat{\partial}_{\alpha}+s^{\alpha} \bar{\partial} r_{\alpha}\right)
$$

where

$$
S_{0}=\int d^{2} z\left(\frac{1}{2} \partial X^{a} \bar{\partial} X_{a}+p_{\alpha} \bar{\partial} \theta^{\alpha}+\omega_{\alpha} \bar{\partial} \lambda^{\alpha}+\xi^{R} \partial \xi^{R}\right)
$$

is the minimal pure spinor action. 
The quantization of this system is performed by introducing a left-moving BRST charge given by

$$
Q=\oint d z\left(\lambda^{\alpha} d_{\alpha}+\widehat{\omega}^{\alpha} r_{\alpha}\right)
$$

where

$$
d_{\alpha}=p_{\alpha}-\frac{1}{2}\left(\theta \gamma^{a}\right)_{\alpha}\left(\partial X_{a}+\frac{1}{4}\left(\theta \gamma_{a} \partial \theta\right)\right)
$$

This BRST charge is nilpotent because of the OPE

$$
d_{\alpha}(y) d_{\beta}(z) \rightarrow-\frac{1}{(y-z)} \gamma_{\alpha \beta}^{a} \Pi_{a}(z)
$$

where

$$
\Pi_{a}=\partial X_{a}+\frac{1}{2}\left(\theta \gamma_{a} \partial \theta\right) .
$$

The BRST transformations of the worldsheet fields of our system are

$$
\begin{aligned}
& Q \Pi^{a}=-\left(\lambda \gamma^{a} \partial \theta\right), \quad Q \theta^{\alpha}=\lambda^{\alpha}, \quad Q d_{\alpha}=\Pi^{a}\left(\gamma_{a} \lambda\right)_{\alpha}, \quad Q \lambda^{\alpha}=0, \quad Q \omega_{\alpha}=d_{\alpha}, \\
& Q \widehat{\lambda}_{\alpha}=-r_{\alpha}, \quad Q \widehat{\omega}^{\alpha}=0, \quad Q r_{\alpha}=0, \quad Q s^{\alpha}=\widehat{\omega}^{\alpha} .
\end{aligned}
$$

Note that the non-minimal sector in our system does not change the cohomology of the minimal sector and the action (2.4) can be written as

$$
S=S_{0}+Q \int d^{2} z s^{\alpha} \widehat{\partial}_{\alpha}
$$

The non-minimal pure spinor formalism does not contain the $(b, c)$ worldsheet reparameterization ghosts. However, one can construct an operator $b$ satisfying the equation $Q b=T$ where $T$ is the world-sheet stress tensor of the action (2.4), and this operator is identified with the pure spinor $b$ ghost [2]. It was shown in [3] that this $b$ ghost is simplified by introducing the RNS-like fermionic vector

$$
\bar{\Gamma}_{a}=-\frac{1}{2(\lambda \hat{\lambda})}\left(d \gamma_{a} \hat{\lambda}\right)-\frac{1}{8(\lambda \hat{\lambda})^{2}}\left(r \gamma_{a b c} \hat{\lambda}\right) N^{b c}
$$

where $N^{a b}=\frac{1}{2}\left(\lambda \gamma^{a b} \omega\right)$ is the Lorentz current for the minimal pure spinors.

In terms of (2.12), the pure spinor $b$ ghost is

$$
b=-s^{\alpha} \partial \widehat{\lambda}_{\alpha}-\omega_{\alpha} \partial \theta^{\alpha}+\Pi^{a} \bar{\Gamma}_{a}-\frac{1}{4(\lambda \widehat{\lambda})}\left(\lambda \gamma^{a b} r\right) \bar{\Gamma}_{a} \bar{\Gamma}_{b}+\frac{1}{2(\lambda \widehat{\lambda})}\left(\omega \gamma_{a} \widehat{\lambda}\right)\left(\lambda \gamma^{a} \partial \theta\right) .
$$

To verify the relation $Q b=T$, we first compute the action of $Q$ on $\bar{\Gamma}_{a}$ to be

$$
Q \bar{\Gamma}_{a}=-\frac{1}{2(\lambda \hat{\lambda})} \Pi^{b}\left(\widehat{\lambda} \gamma_{a} \gamma_{b} \lambda\right)-\frac{1}{4(\lambda \hat{\lambda})^{2}}\left(\lambda \gamma_{b c} r\right)\left(\widehat{\lambda} \gamma^{c} \gamma_{a} \lambda\right) \bar{\Gamma}^{b}
$$

The proof of (2.14) and the verification of $Q b=T$ in a flat background are in the appendix.

The purpose of this paper is to find a $\bar{\Gamma}_{a}$ in a curved heterotic superstring background that satisfies an equation analogous to (2.14) and to define the $b$ ghost as the covariantization of (2.13). In order to do this, we will need the BRST transformations corresponding to (2.10) in a curved background. We now review the non-minimal pure spinor formalism in a curved heterotic superstring background. 


\subsection{Non-minimal pure spinor formalism in a curved background}

The minimal sector of the heterotic string in a curved background was constructed in [6]. The action has the form

$$
S_{0}=\int d^{2} z\left(\frac{1}{2} \Pi_{a} \bar{\Pi}^{a}+\frac{1}{2} \Pi^{A} \bar{\Pi}^{B} B_{B A}+d_{\alpha} \bar{\Pi}^{\alpha}+\omega_{\alpha} \bar{\nabla} \lambda^{\alpha}+\xi^{R} \nabla \xi^{R}+\frac{1}{2} \alpha^{\prime} \Phi r\right),
$$

where $\Pi^{A}$ and $\bar{\Pi}^{A}$ for $A=(a, \alpha)$ are defined from the background supervielbein $E_{M}{ }^{A}$ and the target superspace coordinates $Z^{M}$ as $\Pi^{A}=\partial Z^{M} E_{M}{ }^{A}$ and $\bar{\Pi}^{A}=\bar{\partial} Z^{M} E_{M}{ }^{A}, B_{B A}$ is the graded-antisymmetric two-form superfield, $\Phi$ is the dilaton superfield which couples to the two-dimensional worldsheet curvature $r$,

$$
\nabla \xi^{R}=\partial \xi^{R}+T_{I}^{R S} \xi^{S}\left(\Pi^{A} A_{A}^{I}+d_{\alpha} W^{I \alpha}+\frac{1}{2} N_{a b} F^{I a b}\right)
$$

where $T_{I}^{R S}$ are the $\mathrm{SO}(32)$ adjoint matrices for $I=1$ to 496 and $\left(A_{M}^{I}, W^{I \alpha}, F^{I a b}\right)$ are the super-Yang-Mills gauge fields and field-strengths, and

$$
\bar{\nabla} \lambda^{\alpha}=\bar{\partial} \lambda^{\alpha}+\lambda^{\beta} \bar{\Pi}^{A} \Omega_{A \beta}{ }^{\alpha}
$$

where the connection $\Omega_{A \beta}{ }^{\alpha}$ has the structure

$$
\Omega_{A \beta}{ }^{\alpha}=\Omega_{A} \delta_{\beta}^{\alpha}+\frac{1}{4} \Omega_{A a b}\left(\gamma^{a b}\right)_{\beta}{ }^{\alpha} .
$$

Here $\Omega_{A a b}$ is the usual Lorentz connection and $\Omega_{A}$ is a connection for scaling transformations introduced in [6], and one can verify their coupling preserves the pure spinor gauge invariance of (2.3).

The presence of the scale connection $\Omega_{A}$ in (2.15) implies that the action is invariant not only under the usual local Lorentz transformations, but also under the local fermionic scale transformations

$$
\begin{aligned}
\delta \lambda^{\alpha} & =\Lambda \lambda^{\alpha}, \quad \delta \omega_{\alpha}=-\Lambda \omega_{\alpha}, & \delta d_{\alpha} & =-\Lambda d_{\alpha}, \\
\delta \Omega_{\alpha \beta}{ }^{\gamma} & =-\left(\partial_{\alpha} \Lambda\right) \delta_{\beta}^{\gamma}-\Lambda \Omega_{\alpha \beta}{ }^{\gamma}, & \delta \Omega_{a \beta}{ }^{\gamma} & =-\left(\partial_{a} \Lambda\right) \delta_{\beta}^{\gamma}, \\
\delta E_{M}{ }^{\alpha} & =\Lambda E_{M}{ }^{\alpha}, & \delta E_{\alpha}{ }^{M} & =-\Lambda E_{\alpha}{ }^{M} .
\end{aligned}
$$

So variables and superfields with raised tangent-space spinor indices carry charge +1 with respect to the fermionic scale transformations and variables and superfields with lowered tangent-space spinor indices carry charge -1 .

The minimal BRST charge is given by $Q_{0}=\oint \lambda^{\alpha} d_{\alpha}$ and it was shown in [6] that nilpotency and holomorphicity of $Q_{0}$ forces the background to satisfy the equations of $N=1$ ten-dimensional supergravity. Nilpotency implies that

$$
\lambda^{\alpha} \lambda^{\beta} T_{\alpha \beta}{ }^{A}=0, \quad \lambda^{\alpha} \lambda^{\beta} \lambda^{\gamma} R_{\alpha \beta \gamma}{ }^{\delta}=0,
$$

where $T_{\alpha \beta}{ }^{A}$ and $R_{\alpha \beta \gamma}{ }^{\delta}$ are torsion and curvature components. And as shown in [6], nilpotency and holomorphicity imply that the torsion components can be gauge-fixed to the form

$$
T_{\alpha \beta}^{a}=\gamma_{\alpha \beta}^{a}, \quad T_{A \beta}^{\gamma}=0, \quad T_{\alpha a}^{b}=2\left(\gamma_{a}^{b}\right)_{\alpha}^{\beta} \Omega_{\beta}
$$


In addition, the absence of chiral [6] and conformal [7] anomalies of the worldsheet action implies that $\Omega_{\alpha}$ is related to the dilaton superfield $\Phi$ by

$$
\Omega_{\alpha}=\frac{1}{4} D_{\alpha} \Phi .
$$

The BRST transformations of the minimal fields in (2.15) were determined in [8] to be

$$
\begin{array}{ll}
Q \Pi^{a}=-\lambda^{\beta} \Omega_{\beta b}{ }^{a} \Pi^{b}-\lambda^{\alpha} \Pi^{b} T_{b \alpha}{ }^{a}, & Q \Pi^{\alpha}=-\lambda^{\beta} \Omega_{\beta \gamma}{ }^{\alpha} \Pi^{\gamma}+\nabla \lambda^{\alpha}, \\
Q \lambda^{\alpha}=-\lambda^{\beta} \Omega_{\beta \gamma}{ }^{\alpha} \lambda^{\gamma}, & Q \omega_{\alpha}=\lambda^{\beta} \Omega_{\beta \alpha}{ }^{\gamma} \omega_{\gamma}+d_{\alpha}, \\
Q d_{\alpha}=\lambda^{\beta} \Omega_{\beta \alpha}{ }^{\gamma} d_{\gamma}+\Pi^{a}\left(\gamma_{a} \lambda\right)_{\alpha}+\lambda^{\beta} \lambda^{\gamma} \omega_{\delta} R_{\alpha \beta \gamma}{ }^{\delta}, &
\end{array}
$$

where the first term in these transformations is a Lorentz and scale transformation proportional to $\lambda^{\beta} \Omega_{\beta \gamma}{ }^{\alpha}$.

For the non-minimal sector, it was noted in [5] that there is an effect of the background geometry on the BRST transformations of the non-minimal pure spinor fields. Assuming that the minimal sector is unaffected by the non-minimal variables, a cohomological argument determined that $(\widehat{\lambda}, \widehat{\omega}, r, s)$ transform in a curved background as

$$
\begin{aligned}
Q \widehat{\lambda}_{\alpha} & =-r_{\alpha}+\lambda^{\gamma} \widehat{\lambda}_{\beta}\left(\Omega_{\gamma \alpha}{ }^{\beta}-\frac{1}{4} T_{\gamma a b}\left(\gamma^{a b}\right)_{\alpha}{ }^{\beta}\right), \\
Q \widehat{\omega}^{\alpha} & =-\widehat{\omega}^{\beta} \lambda^{\gamma}\left(\Omega_{\gamma \beta}{ }^{\alpha}-\frac{1}{4} T_{\gamma a b}\left(\gamma^{a b}\right)_{\beta}{ }^{\alpha}\right), \\
Q s^{\alpha} & =\widehat{\omega}^{\alpha}+s^{\beta} \lambda^{\gamma}\left(\Omega_{\gamma \beta}{ }^{\alpha}-\frac{1}{4} T_{\gamma a b}\left(\gamma^{a b}\right)_{\beta}{ }^{\alpha}\right), \\
Q r_{\alpha} & =-\lambda^{\gamma} r_{\beta}\left(\Omega_{\gamma \alpha}{ }^{\beta}-\frac{1}{4} T_{\gamma a b}\left(\gamma^{a b}\right)_{\alpha}^{\beta}\right) .
\end{aligned}
$$

Note that the torsion $T_{\gamma a b}$ includes the Lorentz connection $\Omega_{\gamma a b}$, so the Lorentz part of the spin connection of (2.18) does not appear in these non-minimal BRST transformations.

To construct the non-minimal action in a curved background, the BRST-trivial term

$$
S_{\text {non-min }}=Q \int d^{2} z\left(s \bar{\nabla} \hat{\lambda}+\frac{1}{4} \bar{\Pi}^{A} T_{A a b}\left(s \gamma^{a b} \hat{\lambda}\right)\right)
$$

will be added to the minimal action of (2.15) where $\bar{\nabla} \hat{\lambda}_{\alpha}=\bar{\partial} \widehat{\lambda}_{\alpha}-\widehat{\lambda}_{\beta} \bar{\Pi}^{A} \Omega_{A \alpha}{ }^{\beta}$. This construction is analogous to the flat action of (2.11), and although the torsion term in (2.25) is not needed for covariance and was not included in [5], it will simplify the construction by decoupling the Lorentz connection $\Omega_{A a b}$ from the non-minimal action. Using the BRST transformations of $(2.24)$, one finds that

$$
\begin{aligned}
S_{\text {non-min }}= & \int d^{2} z\left(\widehat{\omega}^{\alpha} \bar{\nabla} \widehat{\lambda}_{\alpha}+s^{\alpha} \bar{\nabla} r_{\alpha}+\frac{1}{4} \bar{\Pi}^{A} T_{A a b}\left(\widehat{\omega} \gamma^{a b} \widehat{\lambda}+s \gamma^{a b} r\right)\right. \\
& \left.+\lambda^{\alpha} \bar{\Pi}^{A} R_{A \alpha}\left(s^{\beta} \widehat{\lambda}_{\beta}\right)+\frac{1}{4} \lambda^{\alpha} \bar{\Pi}^{A}\left(R_{A \alpha a b}-\nabla_{[A} T_{\alpha] a b}-T_{A \alpha}{ }^{c} T_{c a b}+T_{A c[a} T_{b] \alpha}{ }^{c}\right)\left(s \gamma^{a b} \widehat{\lambda}\right)\right) \\
= & \int d^{2} z\left(\widehat{\omega}^{\alpha} \bar{\nabla} \widehat{\lambda}_{\alpha}+s^{\alpha} \bar{\nabla} r_{\alpha}+\frac{1}{4} \bar{\Pi}^{A} T_{A a b}\left(\widehat{\omega} \gamma^{a b} \widehat{\lambda}+s \gamma^{a b} r\right)\right. \\
& \left.+\lambda^{\alpha} \bar{\Pi}^{A} R_{A \alpha}\left(s^{\beta} \widehat{\lambda}_{\beta}\right)+\frac{1}{4} \lambda^{\alpha} \bar{\Pi}^{d}\left(R_{d \alpha a b}-\nabla_{[d} T_{\alpha] a b}-T_{d \alpha}{ }^{c} T_{c a b}+T_{d c[a} T_{b] \alpha}{ }^{c}\right)\left(s \gamma^{a b} \widehat{\lambda}\right)\right)
\end{aligned}
$$


where we have used the Bianchi identity

$$
R_{\beta \alpha a b}-\nabla_{(\beta} T_{\alpha) a b}-T_{(\beta a}{ }^{c} T_{\alpha) c b}-\gamma_{\beta \alpha}^{c} T_{c a b}=0
$$

in the second line of (2.26).

Using the Noether method, one can easily determine the BRST charge corresponding to the action of $S=S_{0}+S_{\text {non-min }}$ to be

$$
Q=\int d z\left(\lambda^{\alpha} d_{\alpha}+\widehat{\omega}^{\alpha} r_{\alpha}\right)
$$

\section{Definition of $\bar{\Gamma}_{a}$ in curved background}

\subsection{Simplified BRST transformations}

The first step in defining the curved background generalization of $\bar{\Gamma}_{a}$ of $(2.12)$ is to define a new variable

$$
D_{\alpha}=d_{\alpha}+\frac{1}{4} \lambda^{\beta} T_{\beta a b}\left(\gamma^{a b} \omega\right)_{\alpha}-3(\lambda \Omega) \omega_{\alpha} .
$$

In terms of $D_{\alpha}$, the BRST transformation of $\omega_{\alpha}$ is given by

$$
Q \omega_{\alpha}=\lambda^{\beta} \Omega_{\beta \alpha}{ }^{\gamma} \omega_{\gamma}+D_{\alpha}+3(\lambda \Omega) \omega_{\alpha}-\frac{1}{4} \lambda^{\beta} T_{\beta a b}\left(\gamma^{a b} \omega\right)_{\alpha}
$$

Furthermore, the BRST transformation of $D_{\alpha}$ is

$$
\begin{aligned}
Q D_{\alpha}= & \lambda^{\beta} \Omega_{\beta \alpha}{ }^{\gamma} D_{\gamma}+\Pi^{a}\left(\gamma_{a} \lambda\right)_{\alpha}+3(\lambda \Omega) D_{\alpha}-\frac{1}{4} \lambda^{\beta} T_{\beta a b}\left(\gamma^{a b} D\right)_{\alpha} \\
& +\lambda^{\beta} \lambda^{\gamma} \omega_{\delta}\left(R_{\alpha \beta \gamma}{ }^{\delta}+\frac{1}{4}\left(\gamma^{a b}\right)_{\alpha}{ }^{\delta} \nabla_{\gamma} T_{\beta a b}+\frac{1}{16}\left(\gamma^{a b} \gamma^{c d}\right)_{\alpha}{ }^{\delta} T_{\beta a b} T_{\gamma c d}\right) \\
= & \lambda^{\beta} \Omega_{\beta \alpha}{ }^{\gamma} D_{\gamma}+\Pi^{a}\left(\gamma_{a} \lambda\right)_{\alpha}+3(\lambda \Omega) D_{\alpha}-\frac{1}{4} \lambda^{\beta} T_{\beta a b}\left(\gamma^{a b} D\right)_{\alpha}
\end{aligned}
$$

where we have used that $Q(\lambda \Omega)=0$ because $\Omega_{\alpha}$ is proportional to $\nabla_{\alpha} \Phi$. To prove that the second line in (3.3) is zero, symmetrize in $(\beta \gamma)$ and use the Bianchi identity $R_{(\alpha \beta \gamma)}{ }^{\delta}=0$ and $\lambda^{\beta} \lambda^{\gamma} R_{\beta \gamma}=0$ to show that the second line is equal to

$$
\begin{aligned}
& \frac{1}{8} \lambda^{\beta} \lambda^{\gamma} \omega_{\delta}\left(\left(\gamma^{a b}\right)_{\alpha}{ }^{\delta}\left(-R_{\beta \gamma a b}+\nabla_{(\beta} T_{\gamma) a b}\right)+\frac{1}{4}\left[\gamma^{a b}, \gamma^{c d}\right]_{\alpha}{ }^{\delta} T_{\beta a b} T_{\gamma c d}\right) \\
& \quad=\frac{1}{8} \lambda^{\beta} \lambda^{\gamma} \omega_{\delta}\left(\nabla_{(\beta} T_{\gamma) a b}-T_{a(\beta}{ }^{c} T_{\gamma) c b}-R_{\beta \gamma a b}\right)\left(\gamma^{a b}\right)_{\alpha}{ }^{\delta}=-\frac{1}{8} \lambda^{\beta} \lambda^{\gamma} \omega_{\delta} \gamma_{\beta \gamma}^{c} T_{c a b}\left(\gamma^{a b}\right)_{\alpha}{ }^{\delta}=0,
\end{aligned}
$$

where we used the Bianchi identity for $R_{[\beta \gamma a] b}$.

The BRST transformations of (2.24), (3.2) and (3.3) all involve a Lorentz and scale transformation proportional to

$$
-\lambda^{\beta} \Omega_{\beta \alpha}{ }^{\gamma}+\frac{1}{4} \lambda^{\beta} T_{\beta a b}\left(\gamma^{a b}\right)_{\alpha}^{\gamma}
$$


It will be useful to define $\tilde{Q}=Q-Q_{L+S}$ where $Q_{L+S}$ is this Lorentz and scale transformation, and one finds that

$$
\begin{aligned}
& \tilde{Q} \Pi^{a}=-\left(\lambda \gamma^{a} \Pi\right), \quad \tilde{Q} \Pi^{\alpha}=\nabla \lambda^{\alpha}+\frac{1}{4} \lambda^{\beta} T_{\beta a b}\left(\gamma^{a b} \Pi\right)^{\alpha}, \quad \tilde{Q} D_{\alpha}=\Pi^{a}\left(\gamma_{a} \lambda\right)_{\alpha}+3(\lambda \Omega) D_{\alpha}, \\
& \tilde{Q} \lambda^{\alpha}=5(\lambda \Omega) \lambda^{\alpha}, \quad \tilde{Q} \omega_{\alpha}=D_{\alpha}+3(\lambda \Omega) \omega_{\alpha}, \\
& \tilde{Q} \widehat{\lambda}_{\alpha}=-r_{\alpha}, \quad \tilde{Q} \widehat{\omega}^{\alpha}=0, \quad \tilde{Q} r_{\alpha}=0, \quad \tilde{Q} s^{\alpha}=\widehat{\omega}^{\alpha},
\end{aligned}
$$

where we have used that

$$
\tilde{Q} \lambda^{\alpha}=\frac{1}{4} \lambda^{\beta} T_{\beta a b}\left(\gamma^{a b} \lambda\right)^{\alpha}=\frac{1}{2}\left(\lambda \gamma_{a b} \Omega\right)\left(\gamma^{a b} \lambda\right)^{\alpha}=5(\lambda \Omega) \lambda^{\alpha} .
$$

\subsection{Construction of $\bar{\Gamma}_{a}$}

In this subsection, it will be shown that

$$
\bar{\Gamma}_{a}=-\frac{1}{2(\lambda \hat{\lambda})}\left(D \gamma_{a} \widehat{\lambda}\right)-\frac{1}{8(\lambda \hat{\lambda})^{2}}\left(r \gamma_{a b c} \widehat{\lambda}\right) N^{b c}
$$

satisfies the BRST transformation

$$
\tilde{Q} \bar{\Gamma}_{a}=-\frac{1}{2(\lambda \hat{\lambda})} \Pi^{b}\left(\hat{\lambda} \gamma_{a} \gamma_{b} \lambda\right)-\frac{1}{4(\lambda \hat{\lambda})^{2}}\left(\lambda \gamma_{b c} r\right)\left(\hat{\lambda} \gamma^{c} \gamma_{a} \lambda\right) \bar{\Gamma}^{a}-2\left(\lambda^{\alpha} \Omega_{\alpha}\right) \bar{\Gamma}_{a}
$$

where $\tilde{Q}$ is defined in (3.5). Comparing with the equations of (2.12) and (2.14), one sees that (3.7) is constructed in a curved background by replacing $d_{\alpha}$ in the flat-space construction with $D_{\alpha}$ of (3.1).

Since the BRST transformations of (3.5) closely resemble the flat space BRST transformation of (2.10), the only necessary step to proving (3.8) is to show that the terms $\left(\lambda^{\alpha} \Omega_{\alpha}\right)$ which appear in (3.5) sum up to $-2\left(\lambda^{\alpha} \Omega_{\alpha}\right) \bar{\Gamma}_{\alpha}$. From the first term in (3.7), one obtains

$$
\frac{1}{2(\lambda \hat{\lambda})^{2}}(5(\lambda \widehat{\lambda})(\lambda \Omega))\left(D \gamma_{a} \widehat{\lambda}\right)-\frac{1}{2(\lambda \hat{\lambda})}\left(3(\lambda \Omega) D_{\alpha}\right)\left(\gamma_{a} \widehat{\lambda}\right)^{\alpha}=2(\lambda \Omega) \frac{1}{2(\lambda \hat{\lambda})}\left(D \gamma_{a} \widehat{\lambda}\right)
$$

where the first term comes from the transformation of $(\lambda \widehat{\lambda})^{-1}$ and the second term comes from the transformation of $D_{\alpha}$. And from the second term in (3.7), one obtains

$$
\begin{gathered}
\frac{1}{4(\lambda \hat{\lambda})^{3}}(5(\lambda \widehat{\lambda})(\lambda \Omega))\left(r \gamma_{a b c} \widehat{\lambda}\right) N^{b c}+\frac{1}{8(\lambda \widehat{\lambda})^{2}}\left(r \gamma_{a b c} \widehat{\lambda}\right)\left(5(\lambda \Omega) N^{b c}+3(\lambda \Omega) N^{b c}\right) \\
=2(\lambda \Omega) \frac{1}{8(\lambda \widehat{\lambda})^{2}}\left(r \gamma_{a b c} \widehat{\lambda}\right) N^{b c}
\end{gathered}
$$

where the first term comes from the transformation of $(\lambda \widehat{\lambda})^{-1}$ and the second term comes from the transformation of $N^{b c}$. So we have proven (3.8). 


\section{Definition of $b$ ghost in curved background}

In this section, we will use the dynamical twisting method of [3] to simplify the construction of the $b$ ghost in a curved heterotic background which was proposed in [5]. We will show that the $b$ ghost in a curved background can be defined in terms of the dynamically twisted RNS-like variable (3.7) by simply covariantizing the flat-space expression of (2.13) as

$b=-s^{\alpha} \nabla \widehat{\lambda}_{\alpha}+\frac{1}{4} \Pi^{A} T_{A a b}\left(s \gamma^{a b} \widehat{\lambda}\right)-\omega_{\alpha} \Pi^{\alpha}+\Pi^{a} \bar{\Gamma}_{a}-\frac{1}{4(\lambda \hat{\lambda})}\left(\lambda \gamma^{a b} r\right) \bar{\Gamma}_{a} \bar{\Gamma}_{b}+\frac{1}{2(\lambda \hat{\lambda})}\left(\omega \gamma_{a} \widehat{\lambda}\right)\left(\lambda \gamma^{a} \Pi\right)$.

As in the action of (2.25), the torsion term in (4.1) is not needed for covariance and was not included in [5], but simplifies the construction by removing the dependence of the $b$ ghost on the Lorentz connection $\Omega_{A a b}$.

To prove that $Q b=T$ where $T$ is the stress-energy tensor of the heterotic string in a curved background, note that $S=S_{0}+S_{\text {non-min }}$ of (2.15) and (2.25) implies that

$$
T=-\frac{1}{2} \Pi_{a} \Pi^{a}-d_{\alpha} \Pi^{\alpha}-\omega_{\alpha} \nabla \lambda^{\alpha}+Q\left(-s^{\alpha} \nabla \widehat{\lambda}_{\alpha}+\frac{1}{4} \Pi^{A} T_{A a b}\left(s \gamma^{a b} \widehat{\lambda}\right)\right) .
$$

So one needs to show that

$$
Q b_{\min }=-\frac{1}{2} \Pi_{a} \Pi^{a}-d_{\alpha} \Pi^{\alpha}-\omega_{\alpha} \nabla \lambda^{\alpha}
$$

where

$$
b_{\min }=-\omega_{\alpha} \Pi^{\alpha}+\Pi^{a} \bar{\Gamma}_{a}-\frac{1}{4(\lambda \hat{\lambda})}\left(\lambda \gamma^{a b} r\right) \bar{\Gamma}_{a} \bar{\Gamma}_{b}+\frac{1}{2(\lambda \hat{\lambda})}\left(\omega \gamma_{a} \widehat{\lambda}\right)\left(\lambda \gamma^{a} \Pi\right) .
$$

Although $b_{\min }$ is invariant under local Lorentz transformations, it transforms under the local scale transformation of (2.19) as

$$
\delta b_{\min }=-2 \Lambda \Pi^{a} \bar{\Gamma}_{a}+4 \Lambda \frac{1}{4(\lambda \widehat{\lambda})}\left(\lambda \gamma^{a b} r\right) \bar{\Gamma}_{a} \bar{\Gamma}_{b}
$$

where we have used that $\bar{\Gamma}_{a}$ of (3.7) transforms as $\delta \bar{\Gamma}_{a}=-2 \Lambda \bar{\Gamma}_{a}$. Using the definition of $\tilde{Q}=$ $Q-Q_{L+S}$ in (3.5), (4.3) is therefore implied if

$$
\tilde{Q} b_{\min }+2(\lambda \Omega) \Pi^{a} \bar{\Gamma}_{a}-\frac{(\lambda \Omega)}{(\lambda \hat{\lambda})}\left(\lambda \gamma^{a b} r\right) \bar{\Gamma}_{a} \bar{\Gamma}_{b}=-\frac{1}{2} \Pi_{a} \Pi^{a}-d_{\alpha} \Pi^{\alpha}-\omega_{\alpha} \nabla \lambda^{\alpha} .
$$

Because of the similarity of (3.5) with the flat space BRST transformations of (2.10) and the result that $Q_{\text {flat }} b_{\text {flat }}=T_{\text {flat }}$, proving (4.6) only requires showing that the various factors of $\left(\lambda^{\alpha} \Omega_{\alpha}\right)$ coming from (3.5) and (3.8) cancel out in (4.6).

The first term in (4.4) contributes no factors of $\left(\lambda^{\alpha} \Omega_{\alpha}\right)$ and the second term in (4.4) contributes $-2(\lambda \Omega) \Pi^{a} \bar{\Gamma}_{a}$ from the $\tilde{Q}$ variation of $\bar{\Gamma}_{a}$. The third term in (4.4) contributes

$$
\begin{aligned}
\frac{1}{4(\lambda \widehat{\lambda})^{2}}(5 & (\lambda \widehat{\lambda})(\lambda \Omega))\left(\lambda \gamma^{a b} r\right) \bar{\Gamma}_{a} \bar{\Gamma}_{b}-\frac{1}{4(\lambda \hat{\lambda})}\left(5(\lambda \Omega) \lambda^{\alpha}\right)\left(\gamma^{a b} r\right)_{\alpha} \bar{\Gamma}_{a} \bar{\Gamma}_{b}+\frac{(\lambda \Omega)}{(\lambda \hat{\lambda})}\left(\lambda \gamma^{a b} r\right) \bar{\Gamma}_{a} \bar{\Gamma}_{b} \\
= & \frac{(\lambda \Omega)}{(\lambda \widehat{\lambda})}\left(\lambda \gamma^{a b} r\right) \bar{\Gamma}_{a} \bar{\Gamma}_{b}
\end{aligned}
$$


where the first term comes from the variation of $(\lambda \widehat{\lambda})^{-1}$, the second term from the variation of $\lambda^{\alpha}$, and the third term from the variation of $\bar{\Gamma}_{a} \bar{\Gamma}_{b}$. Finally, the fourth term in (4.4) contributes

$$
\begin{aligned}
& -\frac{1}{2(\lambda \hat{\lambda})^{2}}(5(\lambda \Omega)(\lambda \hat{\lambda}))\left(\omega \gamma_{a} \widehat{\lambda}\right)\left(\lambda \gamma^{a} \Pi\right)+\frac{1}{2(\lambda \hat{\lambda})} 3(\lambda \Omega)\left(\omega \gamma_{a} \widehat{\lambda}\right)\left(\lambda \gamma^{a} \Pi\right) \\
& \quad+\frac{1}{2(\lambda \hat{\lambda})}\left(\omega \gamma_{a} \hat{\lambda}\right) 5(\lambda \Omega)\left(\lambda \gamma^{a} \Pi\right)+\frac{1}{2(\lambda \hat{\lambda})}\left(\omega \gamma_{a} \widehat{\lambda}\right)\left(\lambda \gamma^{a}\right)_{\alpha} \frac{1}{4} \lambda^{\beta} T_{\beta b c}\left(\gamma^{b c} \Pi\right)^{\alpha}=0
\end{aligned}
$$

where the first term comes from the transformation of $(\lambda \widehat{\lambda})^{-1}$, the second term comes from the transformation of $\omega_{\alpha}$, the third term comes from the transformation of $\lambda^{\alpha}$, and the last term comes from the transformation of $\Pi^{\alpha}$. To show that (4.8) is zero, we have used that

$$
\begin{aligned}
\frac{1}{4(\lambda \hat{\lambda})}\left(\lambda \gamma_{b c} \Omega\right)\left(\lambda \gamma_{a} \gamma_{b c} \Pi\right)\left(\omega \gamma^{a} \widehat{\lambda}\right) & =\frac{1}{4(\lambda \hat{\lambda})}\left(\lambda \gamma_{b c} \Omega\right)\left(\lambda\left(\left[\gamma_{a}, \gamma_{b c}\right]+\gamma_{b c} \gamma_{a}\right) \Pi\right)\left(\omega \gamma^{a} \widehat{\lambda}\right) \\
& =\frac{1}{(\lambda \widehat{\lambda})}\left(\lambda \gamma_{a c} \Omega\right)\left(\lambda \gamma^{c} \Pi\right)\left(\omega \gamma^{a} \widehat{\lambda}\right)+\frac{1}{4(\lambda \hat{\lambda})}\left(\lambda \gamma_{b c} \Omega\right)\left(\lambda \gamma_{b c} \gamma_{a} \Pi\right)\left(\omega \gamma^{a} \widehat{\lambda}\right) \\
& =-\frac{3}{2(\lambda \hat{\lambda})}(\lambda \Omega)\left(\lambda \gamma_{a} \Pi\right)\left(\omega \gamma^{a} \widehat{\lambda}\right) .
\end{aligned}
$$

So we have proven that the $(\lambda \Omega)$ factors cancel out in (4.6), and therefore $Q b=T$.

\section{Acknowledgments}

NB would like to thank Sebastian Guttenberg and Luca Mazzucato for useful discussions. The work of NB is partially financed by CNPq grant 300256/94-9 and FAPESP grants 2009/50639-2 and 2011/11973-4, and the work of OC is partially financed by FONDECYT project 1120263.

\section{A Computations in a flat background}

In this appendix, we will prove the equation (2.14) for $Q \bar{\Gamma}_{a}$ and the equation $Q b=T$ in a flat background.

Using the BRST transformations of (2.10) acting on $\bar{\Gamma}_{a}$ of (2.12), one finds

$$
\begin{aligned}
Q \bar{\Gamma}_{a}= & -\frac{1}{2(\lambda \hat{\lambda})} \Pi^{b}\left(\widehat{\lambda} \gamma_{a} \gamma_{b} \lambda\right)-\frac{1}{2(\lambda \hat{\lambda})^{2}}(\lambda r)\left(d \gamma_{a} \widehat{\lambda}\right)-\frac{1}{2(\lambda \hat{\lambda})}\left(d \gamma_{a} r\right) \\
& -\frac{1}{4(\lambda \hat{\lambda})^{3}}(\lambda r)\left(r \gamma_{a b c} \widehat{\lambda}\right) N^{b c}-\frac{1}{8(\lambda \hat{\lambda})^{2}}\left(r \gamma_{a b c} r\right) N^{b c}+\frac{1}{16(\lambda \hat{\lambda})}\left(r \gamma_{a b c} \widehat{\lambda}\right)\left(\lambda \gamma^{b c} d\right) .
\end{aligned}
$$

The term independent of $r$ agrees in (2.14) and (A.1), and the term linear in $r$ in (A.1) is

$$
\begin{aligned}
- & \frac{1}{2(\lambda \hat{\lambda})^{2}}(\lambda r)\left(d \gamma_{a} \widehat{\lambda}\right)-\frac{1}{2(\lambda \hat{\lambda})}\left(d \gamma_{a} r\right)+\frac{1}{16(\lambda \widehat{\lambda})^{2}}\left(r \gamma_{a} \gamma_{b c} \widehat{\lambda}\right)\left(\lambda \gamma^{b c} d\right) \\
= & -\frac{1}{2(\lambda \hat{\lambda})^{2}}(\lambda r)\left(d \gamma_{a} \widehat{\lambda}\right)+\frac{1}{4(\lambda \widehat{\lambda})^{2}}\left(\lambda \gamma_{b} \gamma_{a} r\right)\left(d \gamma^{b} \widehat{\lambda}\right) \\
& =-\frac{1}{4(\lambda \hat{\lambda})^{2}}\left(\lambda \gamma_{a} \gamma_{b} r\right)\left(d \gamma^{b} \widehat{\lambda}\right)=\frac{1}{8(\lambda \widehat{\lambda})^{3}}\left(\lambda \gamma_{b c} r\right)\left(\widehat{\lambda} \gamma^{c} \gamma_{a} \lambda\right)\left(d \gamma^{b} \widehat{\lambda}\right)
\end{aligned}
$$


which is the term linear in $r$ in (2.14). To go from the first line to the second line of (A.2), we have used the identity

$$
\frac{1}{2} \delta_{\alpha}^{\delta} \delta_{\beta}^{\gamma}+\frac{1}{16}\left(\gamma^{b c}\right)_{\alpha}^{\gamma}\left(\gamma_{b c}\right)_{\beta}{ }^{\delta}=\frac{1}{4} \gamma_{\alpha \beta}^{b} \gamma_{b}^{\gamma \delta}-\frac{1}{8} \delta_{\alpha}^{\gamma} \delta_{\beta}^{\delta}
$$

together with the pure spinor constraints of (2.1).

Finally, the terms quadratic in $r$ in (A.1) are

$$
\begin{aligned}
& -\frac{1}{8(\lambda \hat{\lambda})^{2}}\left(r \gamma_{a b c} r\right) N^{b c}-\frac{1}{4(\lambda \hat{\lambda})^{3}}(\lambda r)\left(r \gamma_{a b c} \widehat{\lambda}\right) N^{b c} \\
& =-\frac{1}{8(\lambda \hat{\lambda})^{2}}\left(r \gamma_{a b c} r\right) N^{b c}-\frac{1}{384(\lambda \hat{\lambda})^{3}}\left(\lambda \gamma_{d e f} \gamma_{b c} \gamma_{a} \widehat{\lambda}\right)\left(r \gamma^{d e f} r\right) N^{b c} \\
& =-\frac{1}{8(\lambda \hat{\lambda})^{2}}\left(r \gamma_{a b c} r\right) N^{b c}+\frac{1}{16(\lambda \hat{\lambda})^{3}}\left(\lambda \gamma_{b} \gamma_{a} \widehat{\lambda}\right)\left(r \gamma^{b d e} r\right) N_{d e} \\
& =-\frac{1}{16(\lambda \hat{\lambda})^{3}}\left(\lambda \gamma_{a} \gamma_{b} r\right)\left(r \gamma^{b d e} \hat{\lambda}\right) N_{d e},
\end{aligned}
$$

which is the term quadratic in $r$ in (2.14). To go from the first line to the second line of (A.4), we have used the identity $r_{\alpha} r_{\beta}=\frac{1}{96} \gamma_{\alpha \beta}^{\text {def }}\left(r \gamma_{d e f} r\right)$. To go from the second line to the third line, we have used that $\left(\lambda \gamma^{b}\right)_{\alpha} N_{b c}=\frac{1}{2} J\left(\gamma^{c} \lambda\right)_{\alpha}$ where $J=-\lambda^{\alpha} \omega_{\alpha}$ and that all terms proportional to $J$ vanish using the pure spinor constraints of (2.1). And to go from the third line to the fourth line, we have used that $\left(\gamma_{b} r\right)^{\alpha}\left(\gamma^{b} \widehat{\lambda}\right)^{\beta}=-\left(\gamma_{b} \widehat{\lambda}\right)^{\alpha}\left(\gamma^{b} r\right)^{\beta}$. So we have proven that $\bar{\Gamma}_{a}$ satisfies equation (2.14) in a flat background.

We now verify that $Q b=T$ in flat space. Applying $Q$ of (2.10) to (2.13), we obtain

$$
\begin{aligned}
Q b= & T+\left(\lambda \gamma^{a} \Pi\right)\left(\frac{1}{8(\lambda \hat{\lambda})^{2}}\left(r \gamma_{a b c} \widehat{\lambda}\right) N^{b c}-\frac{1}{2(\lambda \widehat{\lambda})^{2}}(\lambda r)\left(\omega \gamma_{a} \widehat{\lambda}\right)+\frac{1}{2(\lambda \hat{\lambda})}\left(\omega \gamma_{a} r\right)\right) \\
& -\frac{1}{4(\lambda \hat{\lambda})^{2}} \bar{\Gamma}^{a} \bar{\Gamma}^{b}\left((\lambda r)\left(\lambda \gamma_{a b} r\right)+\frac{1}{2(\lambda \hat{\lambda})}\left(\lambda \gamma_{a}^{c} r\right)\left(\lambda \gamma_{b d} r\right)\left(\widehat{\lambda} \gamma^{d} \gamma_{c} \lambda\right)\right) .
\end{aligned}
$$

Using the identity

$$
(\lambda \widehat{\lambda})\left(\omega \gamma_{a} r\right)-(\lambda r)\left(\omega \gamma_{a} \widehat{\lambda}\right)=-\frac{1}{8}\left(\widehat{\lambda} \gamma_{a b c} r\right) N^{b c}-\frac{1}{48}\left(\lambda \gamma_{a b c d} \omega\right)\left(r \gamma^{b c d} \widehat{\lambda}\right)
$$

we obtain that the second term in (A.5) is equal to

$$
\frac{1}{16(\lambda \widehat{\lambda})}\left(\lambda \gamma^{a} \Pi\right)\left(\left(r \gamma_{a b c} \widehat{\lambda}\right) N^{b c}+\frac{1}{6}\left(\lambda \gamma_{a b c d} \omega\right)\left(r \gamma^{b c d} \widehat{\lambda}\right)\right)
$$

which can be seen to vanish using $\left(\lambda \gamma^{a}\right)_{\alpha}\left(\lambda \gamma_{a}\right)_{\beta}=0$. Finally, the term proportional to $\bar{\Gamma}^{a} \bar{\Gamma}^{b}$ in (A.5) vanishes using the identities $\left(\lambda \gamma^{a}\right)_{\alpha}\left(\lambda \gamma_{a}\right)_{\beta}=(\lambda r)(\lambda r)=0$.

Open Access. This article is distributed under the terms of the Creative Commons Attribution License (CC-BY 4.0), which permits any use, distribution and reproduction in any medium, provided the original author(s) and source are credited. 


\section{References}

[1] N. Berkovits, Super Poincaré covariant quantization of the superstring, JHEP 04 (2000) 018 [hep-th/0001035] [INSPIRE].

[2] N. Berkovits, Pure spinor formalism as an $N=2$ topological string, JHEP 10 (2005) 089 [hep-th/0509120] [INSPIRE].

[3] N. Berkovits, Dynamical twisting and the $b$ ghost in the pure spinor formalism, JHEP 06 (2013) 091 [arXiv: 1305. 0693] [INSPIRE].

[4] I. Bakhmatov and N. Berkovits, Pure Spinor b-ghost in a Super-Maxwell Background, JHEP 11 (2013) 214 [arXiv:1310.3379] [INSPIRE].

[5] O. Chandía, The non-minimal heterotic pure spinor string in a curved background, JHEP 03 (2014) 095 [arXiv: 1311.7012] [INSPIRE].

[6] N. Berkovits and P.S. Howe, Ten-dimensional supergravity constraints from the pure spinor formalism for the superstring, Nucl. Phys. B 635 (2002) 75 [hep-th/0112160] [INSPIRE].

[7] O. Chandía and B.C. Vallilo, Conformal invariance of the pure spinor superstring in a curved background, JHEP 04 (2004) 041 [hep-th/0401226] [INSPIRE].

[8] O. Chandía, A note on the classical BRST symmetry of the pure spinor string in a curved background, JHEP 07 (2006) 019 [hep-th/0604115] [INSPIRE]. 